JOURNAL REVIEW

\section{Personalized prediction of first-cycle in vitro fertilization success}

Choi B, Bosch E, Lannon BM, et al. Fertil Steril 2013; 20 March. pii: S0015-0282(13)00273-2. doi: 10.1016/j.fertnstert.2013.02.016

This paper provides the background research that resulted in an online predictor for in vitro fertilisation (IVF) success. It is based on the outcomes of $>13000$ treatments from three centres in the USA, Spain and Canada. Intuitively it attempts to provide an answer for the question that all couples ask when considering IVF: Will it work? The National Institute for Health and Clinical Excellence (NICE) recommends that treatment should be provided for those with a predicted live birth rate of at least $10 \%$ so more accurate prediction could be helpful. Self-funded patients would also have more information upon which they can decide if treatment is cost effective for them.
The results showed that $60.1 \%$ of the predicted outcome depends on the variable of age, whereas the remainder depends on the variables of male factor (9.6\%), body mass index (9.5\%), ovarian reserve $(9.5 \%)$ and others each with $<3 \%$ relevance.

There are some problems though. The analysis does not include those who start treatment but do not reach egg collection, usually because they have poor ovarian reserve. Potential patients need to know the predicted outcome before they start, not when they have already had days of medication. The analysis uses follicle-stimulating hormone to predict ovarian reserve, whereas antiMüllerian hormone is now accepted as the standard endocrine predictor. We should also be very cautious about interpreting the absolute predictions that are given. The analysis was of cumulative pregnancies per egg collection, including transfer of frozen embryos.

Will this predictor be useful in the UK? An alternative online predictor that is based on the Human Fertilisation and Embryology Authority database is freely available (http://www.ivfpredict.com) in comparison with the commercially available predictor (http://www.univfy.com). This takes into account $69.7 \%$ of the variables used by Univfy and is based on UK practice. It is unlikely that a few percent difference in prediction will alter decisions. NICE recommendations are based on a $>10 \%$ predicted birth rate so more complex predictions are unlikely to be cost effective and would probably be difficult to implement. From the perspective of the self-funded patient, costs are a major issue. However, using the example in this paper, they are unlikely to decide that a predicted pregnancy rate of $34.1 \%$ (Univfy) in comparison with $26.5 \%$ (age-predicted) would change their decision to have a first treatment.

This is an interesting analysis, but I wouldn't advise patients to pay the fee to register for the prediction.

\section{Reviewed by Alison Murdoch}

Professor, Newcastle Fertility Centre at LIFE, Newcastle upon Tyne, UK; a.p.murdoch@ncl.ac.uk

J Fam Plann Reprod Health Care 2013;39:189.

doi:10.1136/jprhc-2013-100653 